\title{
MUJERES Y ESPACIO PÚBLICO: LAS ASOCIACIONES INDÍGENAS FEMENINAS AYMARAS DE LA CIUDAD DE ARICA
}

\author{
AYMARA WOMEN AND PUBLIC SPACE: THE VALUE ASSIGNED TO \\ INDIGENOUS ASSOCIATIONS FEMALE IN ARICA CITY
}

\author{
María Belén Vásquez Santibáñez* y Ana María Carrasco Gutiérrez*
}

\begin{abstract}
En el presente artículo se busca conocer cuáles son las experiencias que las mujeres aymaras del extremo norte de Chile han adquirido en las asociaciones indígenas en las que participan. Mediante un recorrido histórico de las mujeres en los espacios públicos se pretende profundizar respecto de cómo ha sido su participación en estos escenarios y percibir la posición actual que poseen las mujeres aymaras en dichas organizaciones. Para lograr el propósito mencionado se ha realizado un estudio de carácter etnográfico en donde se abordó la experiencia de mujeres aymaras que participan en las Asociaciones Indígenas femeninas de la ciudad de Arica, utilizándose principalmente entrevistas personales y observación participante como técnicas de recolección de la información.

Palabras claves: Mujeres, asociaciones indígenas, Aymara, espacios públicos.
\end{abstract}

In this article we seek to know what are the experiencies that Aymara women delivered them indigenous associations in which they participate. Based on a historical journey of women in public spaces we seek to deepen on how it has been their participation in these scenarios and perceive the current position possessed by Aymara women in these organizations. To achieve the purpose mentioned, it has made a study of ethnographic character in which the experience of Aymara women participating in women's Indigenous Associations of the city of Arica, mainly used personal interviews and participant observation as techniques for gathering information addressed.

Key words: Women, indigenous associations, Aymara, public space.

\section{Introducción}

En la diversa gama de culturas del mundo se manifiestan ciertos roles diferenciados que restringen e idealizan las aptitudes y capacidades de cada género -femenino y masculino-. Esto hace que su imagen e identidad varíen dependiendo de la cultura, así como también las preconcepciones definidas para ambos.

En la cultura aymara, población indígena ubicada en el norte de Chile, se observa que los diferentes roles asignados tradicionalmente para cada género han adquirido características particulares debido a varios factores, entre los que podemos mencionar los históricos, económicos y territoriales que han influido en el desarrollo de este pueblo indígena. Como consecuencia, los hombres se han presentado tradicionalmente dentro del ámbito público, asumiendo la representación familiar y en donde juega un papel principal el acceso al mercado laboral; a diferencia de las mujeres, que se han mantenido preocupadas, fundamentalmente, de ciertos ámbitos específicos dentro del hogar, como actividades domésticas, el cuidado de los hijos, actividades productivas vinculadas principalmente a la artesanía textil, entre otras, manteniéndolas ajenas a intereses fuera del ámbito privado.

Sin embargo, dentro de la dinámica de cambios históricos en los que se ha visto inmersa la sociedad aymara, destaca un momento particular - por las implicancias que trajo consigo- conocido como la reforma político-administrativa, ocurrida en Chile en 1980. Lo anterior llevó al Estado de Chile a transformar la administración del país, la que se basaba en la concentración y administración del poder en la zona central del territorio nacional. Con esta iniciativa se descentraliza el poder, la economía y la población, canalizando los principales servicios y demandas a las municipalidades y a los gobiernos comunales, y posibilitando una mayor participación de la comunidad organizada en el desarrollo de cada región.

Principalmente, la reforma político-administrativa ha involucrado una participación sistemática de la comunidad, en donde las demandas se presentan por intermedio del Consejo de Desarrollo Regional

* Universidad de Tarapacá, Departamento de Antropología, Arica, Chile. Correo electrónico: belenvasquezs@gmail.com; anycarrasco@gmail.com 
(CODERE) y Consejo de Desarrollo Comunal (CODECO), instancias de representación ciudadana que permiten la participación de la sociedad civil en aquellas decisiones gubernamentales que afectan el desarrollo de la región. Este último, CODECO, se conforma por representantes pertenecientes a asociaciones vecinales, organizaciones de la comunidad, grupos de interés local y administrativos municipales. De esta manera, durante este periodo comienza a manifestarse una efectiva participación social de la población permitiendo un incremento en los últimos años. Además, se debe destacar que esto generó un avance paulatino de la participación femenina, ya que se crean espacios donde ellas -al igual que ocurre con otros sectores sociales vulnerables y discriminados- pueden presentar sus demandas y reivindicar sus derechos en el diseño e implementación de políticas, programas y proyectos de desarrollo en materias que les competen.

En la región de Arica y Parinacota (antigua región de Tarapacá), durante 1980 y 1990, las ONG -que interpelaban e incluían la participación de la mujer como un ente social y activo- permitieron la creación de instancias de participación femenina en ámbitos productivos y económicos. Mediante el establecimiento de organizaciones y agrupaciones femeninas, apoyaban al sustento y soporte económico familiar, desarrollando además objetivos adicionales al ámbito económico, los que se vinculaban con la igualdad de género. De este modo, la participación sociopolítica femenina se promovió fuertemente, aun cuando el fin último fuese principalmente instrumental.

Al crearse y promulgarse el 5 de octubre de 1993, por parte del Estado, la Ley Indígena $\mathrm{N}^{\circ} 19.253$ se generaron nuevas formas de organización reconocidas y promovidas por el Estado por medio de la Corporación Nacional de Desarrollo Indígena (CONADI), así como la Comunidad y Asociación Indígena. Según este decreto, las asociaciones indígenas pueden constituirse por diversos objetivos, en donde es posible realizar actividades educacionales, culturales o económicas, buscando solucionar y cambiar las condiciones de vida de las familias indígenas. De esta forma, las agrupaciones de mujeres aymaras existentes que en un comienzo mantenían un fin productivo se reconvierten en asociaciones indígenas femeninas buscando - por su naturaleza- un objetivo cultural mucho más amplio.

Los resultados de la investigación que se presentan en este artículo buscan un acercamiento acerca de la participación de las mujeres aymaras en el espacio público y la importancia que poseen en sus organizaciones. De esta manera, se pretende conocer ¿cuáles son los motivos por los que las mujeres aymaras forman parte de las asociaciones indígenas?; ¿cuáles son las experiencias que las mujeres aymaras han adquirido en las asociaciones indígenas en las que participan? y ¿cuál es la importancia que las mujeres aymaras le otorgan a sus agrupaciones?

De acuerdo con los objetivos propuestos, la metodología empleada ha sido de tipo cualitativa. La información contenida en este artículo se ha obtenido el 2012 mediante la recolección de información primaria y secundaria. En el primer caso se realizaron entrevistas estructuradas a 20 mujeres aymaras radicadas en la ciudad de Arica, XV Región de Arica y Parinacota, que tuvieran participación en alguna asociación indígena aymara. Respecto de la condición étnica, se utilizó como criterio principal el de autoadscripción, considerándose también identificación de sus pares, lugar de origen y apellidos. Su análisis fue mediante el uso de software MAXQDA.

\section{Mujeres y espacios públicos}

\section{La participación femenina latinoamericana}

Investigaciones realizadas en los últimos treinta años dan cuenta de un importante desarrollo teórico en temáticas vinculadas a la historia de las mujeres latinoamericanas y la posición que han ocupado en los espacios públicos. Existen varios estudios que demuestran cómo el segmento femenino ha pasado por diversas situaciones (invisibilidad de lo público, reclusión en el espacio privado, etc.), ocupando un lugar participativo reconocido y relevante en la actualidad (Cf. Rivera 1993; Moscoso 1995; Vargas 2008; Azaola 2012; Zúñiga 2014).

El desarrollo histórico de América Latina nos presenta un carácter patriarcal y de subordinación hacia las mujeres, en donde instituciones claves como la familia y la Iglesia fueron quienes, mediante sus postulados, las mantuvieron excluidas del ámbito político. En el siglo XIX comienzan a percibirse influencias liberales, sin embargo la Iglesia mantuvo el poder sobre la familia y la educación a lo largo del tiempo. No obstante, con el correr de los años -durante la primera mitad del siglo XX- los gobiernos populistas de distintos países (como Brasil, Argentina, México y Colombia), al tener 
intereses propios como, por ejemplo, la obtención de mayor cantidad de votantes a su favor, aprovechan las demandas que las mujeres organizadas exigen y otorgan la ciudadanía por medio del derecho a voto (Luna 2004).

En 1960, aproximadamente, nace el feminismo latinoamericano -el que cuestiona el feminismo hegemónico-y que se lleva a cabo bajo un contexto cultural en donde el activismo político femenino estaba basado en los roles tradicionales de género (Bidaseca 2010; Bouteldja 2014; Meloni 2012). Aunque se efectúa en pleno liberalismo temprano, basado en la igualdad, aún mantenía las ideas y el poder masculino. Sin embargo, en 1970 la lucha de las mujeres por controlar su reproducción así como el reparto de alimentos para la supervivencia en barrios populares de países latinoamericanos fueron aspectos importantes que pudieron desarrollar y visibilizar. Finalmente, en 1980 los gobiernos democráticos (...) fueron un escenario de clara participación de las organizaciones de mujeres desde su diversidad (...) (Luna 2004:48), provocando en su contexto una nueva visión oficial femenina y de desarrollo.

Durante este proceso se observaron otras influencias teóricas que han intervenido en la posición que hoy ocupan las mujeres en las distintas sociedades (Cf. De Oliveira y Gómez 1989; Scott 1996; Lamas 1999; Molyneux 2001; Aguirre 2003; Fassler 2003; Luna 2004; Montecino 2004; Carrasco 2006). De este modo, surgen enfoques de desarrollo orientados hacia las mujeres que buscan hacerlas partícipes de ellos. Dos orientaciones son las que nos interesa mencionar por los impactos que tuvieron en el segmento femenino. Nos referimos al MED (Mujer en el Desarrollo), que surge en los años 1970, el que enfatizaba estrategias de bienestar y antipobreza. Sin embargo, los resultados poco favorables para las mujeres conllevó a diversas críticas tanto desde las organizaciones femeninas como de la academia misma. Por este motivo es que en los años 1980 aparece el enfoque de Género en el Desarrollo (GED), el que se aparta de la mirada exclusivamente economicista que se venía acuñando para mirar el desarrollo y propone la inclusión del concepto de género, sacando a la luz el carácter social y cultural de las identidades masculinas y femeninas (Fassler 2003).

En Latinoamérica, durante 1990 se comienza a tomar en cuenta al Estado como un espacio de intervención feminista, el que facilitaría la mayor representación de la mujer en sus instituciones y en donde, junto con la ayuda de las ONG feministas, se llevarían a cabo esfuerzos para realizar cambios concretos. No obstante, la participación de las mujeres en el Estado aún cuenta con una institucionalización inadecuada para la realización de cambios reales, en donde juega un rol fundamental el partido político que goza del poder y su corriente ideológica (Molyneux 2001).

Bajo esta mirada, existe gran cantidad de información respecto de la débil participación de las mujeres en las instancias de poder en América Latina (Cf. Moore 1991; De Barbieri 1992; Carrasco 1993a; Hernández 2000; Fassler 2003; Valdés 2004; Ruiz y Bonometti 2010). Se establece que en las políticas públicas solo han existido cambios en reformas liberales (por ejemplo, en los derechos laborales) sin tocar temas específicos relacionados al mundo de lo doméstico o privado destinado a la mujer (Montecino 2004). Por este motivo, aunque por parte de las mujeres se muestren grandes iniciativas para acabar con esta brecha reconocida durante años, la dominación masculina social y política continúa ejerciendo su poder.

\section{La situación histórica de las mujeres en Chile}

En Chile se han desarrollado estudios con perspectiva de género que ayudan al conocimiento de la construcción social y cultural del ser hombre y ser mujer, lo que permite una mayor comprensión de las desigualdades participativas que encontramos entre ambos géneros (Montecino 2004; Valdés 2004). Así también, existen evidencias acerca de los primeros movimientos de mujeres, sus luchas en la educación y participación cívica, sus demandas posteriores y su decadencia por no construir grandes liderazgos (Cf. Gaviola 1988, 1994; Frohman y Valdés 1993; Carrasco 1999). Aportes interesantes han sido los estudios realizados por Gaviola (1998), los que buscan reconstruir la historia de las mujeres en Chile, enfocándose en la organización que han tenido en los espacios tanto públicos como políticos, partiendo por la lucha del derecho a voto.

Los primeros movimientos de mujeres responden a luchas por obtener el acceso a la educación superior y a la participación cívica. En 1875 se lleva a cabo por primera vez su intento de participación política por un grupo de mujeres de San Felipe, las que querían hacer valer su derecho a voto. Tras este incidente, en 1884 se crea un reglamento en donde las mujeres quedan excluidas de la posibilidad de 
ejercer este derecho (Carrasco 2006). Durante 1913, organizaciones de mujeres, como por ejemplo los Centros Femeninos Anticlericales Belén de Sárraga en Iquique y en las oficinas salitreras, logran mejores condiciones para las mujeres y la igualdad ante la ley (Valdés 2004). Entre 1915 y 1924 se crean distintos tipos de instituciones femeninas, así como agrupaciones, clubes, centros, etc., los que discuten la condición de la mujer y realizan acciones y reuniones nacionales e internacionales (Kirkwood 1990: 104). Desde 1930 las mujeres comienzan a cumplir roles profesionales. En 1950 se integran a partidos políticos tras eliminarse las instituciones de mujeres durante la dictadura de Carlos Ibáñez, creándose 10 años más tarde por parte del Estado la institucionalización de las organizaciones comunitarias -incluidos los espacios de mujeres-, los que dan lugar a la participación democrática de todos los sectores sociales (Valdés 2004).

La segunda ola de los movimientos de mujeres y del feminismo fueron los responsables de transformaciones de orden social, político y cultural. En los años 1970, Chile se ubicaba en un periodo en donde se intentaba dejar atrás el único lugar de exclusividad de la mujer en la reproducción, maternidad, hogar y crianza de sus hijos. Al adquirir derechos laborales y poseer mayor cantidad de años de educación, se les permite su participación en el poder legislativo y municipios. Sin embargo, aún permanecían excluidas de otros ámbitos, como el poder judicial, ejecutivo e instituciones (Ibíd.). A partir de 1980 se genera un resurgimiento de los movimientos de mujeres mostrándose dos bandos: las mujeres que se posicionan a favor del gobierno militar y las mujeres que están en contra de la dictadura. Finalmente, en 1990 al producirse el traslado de demandas femeninas de la agenda pública hacia las agendas institucionales, se inician los cambios sociales. Sin embargo, el movimiento de mujeres no logra construir grandes liderazgos, ya que al no poder enfrentar las oportunidades dadas se dificulta su rearticulación (Carrasco 2006).

\section{La mujer indígena en los espacios públicos}

En cuanto a la población indígena, en América Latina y el Caribe existen aproximadamente 50 millones de personas, constituyendo el $10 \%$ del total de la población. Junto con esto, los pueblos indígenas alcanzan a 400 comunidades; en estas, $59 \%$ correspondiente a población femenina $(26,5$ millones de personas) y solo $41 \%$ a población masculina (Calfio y Velasco 2005:2). A pesar de la existencia de estos datos generalizados, la mujer indígena en Latinoamérica no puede ser vista como una categoría homogénea, aunque experimenten características discriminatorias por igual y mantengan demandas similares.

Se sabe que las relaciones de género están basadas en construcciones culturales que se crean para ambos sexos. De este modo, en cada comunidad indígena existen patrones culturales específicos que designan, tanto para los hombres como para las mujeres, diferentes grados de aceptación o prohibición para la participación en el ámbito público (Aguirre 2003). Bonfil (2002:71) y Aguirre (2003:3) plantean que la participación de las mujeres indígenas puede darse de dos maneras diferentes. Una manera tradicional, relacionada a los oficios propios que se le asignan a la mujer en una determinada cultura (como tejedoras, alfareras, tortilleras, parteras, bordadoras, panaderas). En contraste, existe la manera no tradicional en donde las mujeres, al tomar conciencia étnica y de género, se organizan para lograr fines concretos (económicos, culturales, de servicios, etc.).

Por otra parte, es de gran importancia considerar la participación que realizan en sus comienzos las ONG a nivel mundial, ya que logran impulsar y apoyar la participación de las mujeres en el ámbito público. Estas fueron promovidas por movimientos de sectores indígenas feministas e impulsadas por marcos jurídicos internacionales (Calfio y Velasco 2005).

Asimismo, es necesario rescatar que durante 1970 y 1980 se llevaron a cabo varias conferencias mundiales, en México (1975), Copenhague (1980) y Nairobi (1985), las que promovían las políticas de género en el desarrollo buscando la igualdad entre hombres y mujeres e incorporando la problemática dentro de la agenda pública de los diferentes países. Finalmente, en 1995 se aprueba la Plataforma de Acción en Beijing, esta buscaba el mejoramiento de la situación de las mujeres indígenas y otorgaba importancia al desarrollo de análisis con enfoque étnico. Sin embargo, y a pesar de estas grandes instancias de apoyo, sigue manteniéndose la brecha participativa de las mujeres indígenas frente al resto de la población. Datos concretos demuestran que son las mujeres indígenas las que presentan más bajos porcentajes de inserción en el trabajo remunerado, junto con las afrodescendientes, hallándose dentro 
de los sectores de baja productividad. Lo anterior es porque aunque existan posibilidades de empleo para las mujeres, estos son espacios laborales vulnerables, mal pagados y socialmente discriminados. Un espacio que coincide con estas características son las áreas de servicios (netamente domésticos). Claramente, mayores niveles educacionales favorecen la participación de las mujeres en el mercado laboral, mejorando sus oportunidades y posibilidades de empleo (Moore 1991).

Los movimientos indígenas en América Latina piden una reconceptualización del concepto de nación en términos de una homogeneidad étnica, planteando así una ciudadanía diferenciada que reconozca las variaciones culturales. Junto con esto, durante el transcurso de los años las acciones de las mujeres desde las organizaciones han buscado influir en la reforma constitucional, acceder al sistema político y el reconocimiento de sus derechos (Safa 2008). Otros tipos de demandas están enfocadas hacia ámbitos económicos -exigiendo salarios justos e iguales para ambos sexos, y que se reconozcan sus aportes en la economía local y nacional-, de la salud y educación, los que son necesarios y fundamentales para su formación y reconocimiento como tales (Calfio y Velasco 2005). En conclusión, y parafraseando a Hernández:

\section{(...) la demanda de las mujeres indígenas es que se reconozca el carácter multicultural de la nación (...) que incluya la diversidad de voces y procesos contradictorios que dan sentido a la vida de un colectivo humano (...) (Hernández 2000:19-20).}

\section{Las participación de las mujeres aymaras del extremo norte de Chile}

Específicamente, en lo que respecta a la población aymara del extremo norte de Chile, existe gran cantidad de información relacionada con los procesos históricos que ha afectado su sociedad y su cultura, así como el proceso de chilenización, influencia judeocristiana, migración, globalización, entre otras, generando cambios en sus modos y condiciones de vida. Los estudios muestran que las principales transformaciones experimentadas tienen su origen desde mediados del siglo XIX, con la desarticulación de las comunidades históricas campesinas, dando paso a “(...) pequeñas localidades agrarias de los valles y a las comunidades sucesoriales de las tierras de la alta cordillera (...)" (Gundermann, 2003:57). Ya desde mediados del siglo XX se inician procesos de transformaciones y cambios modernizadores basados en el paso de microrregiones campesinas a la regionalización de las sociedades andinas o translocalizadas (Cf. Grebe 1986; González, Tabilo y Venegas 1995; González 1995; 1996; Gunderman 2001; Gavilán 2002; Carrasco y González 2012).

Durante 1970, 1980 y 1990 la cultura aymara se insertó dentro de un movimiento de nuevas dinámicas de desarrollo para el país. El gobierno autoritario y el modelo liberal impuesto llevan a cabo reformas de Estado dando inicio, en 1980, a la creación de municipios en zonas de precordillera y alta cordillera. Esto conllevó al origen de nuevas formas de participación bajo la creación de (...) una política multicultural (1990) y de democratización de la elección de autoridades locales (a partir de 1992) (Gundermann 2003:56). A pesar de lo expuesto, estudios concernientes a esta población indígena nos muestran cómo, en la actualidad, constantemente:

(...) los pueblos andinos se enfrentan a entornos tanto sociales y políticos crecientemente complejos; en donde, por lo demás, la desigualdad social y el balance de poderes es decididamente desfavorable para sus intereses y pretensiones (...) (Gundermann 2001: 92).

Autores como Carrasco (1993; 1999), Gavilán (1993), González (1995) dan a conocer información relacionada con la situación y condición de las mujeres aymaras del norte de Chile. En lo referido específicamente a procesos de cambio y mujeres aymaras, Gavilán (1993) y Carrasco (1998) plantean que, desde sus comienzos, la organización social tradicional aymara no disponía de instancias públicas para las mujeres. Por este motivo han sido las principales encargadas del ámbito doméstico y de actividades tanto productivas como reproductivas, no pudiendo acceder y desarrollarse libremente en espacios públicos que generan poder. Ellas trabajan habitualmente:

(...) como jornaleras en cultivos tradicionales de chacras y hortalizas; desarrollan otras actividades tales como el comercio en pequeña escala e incluso realizan otra importante actividad económica, como lo 
es la producción y comercialización de artesanías (...) (Carrasco 1998:4).

Sin embargo, la presencia femenina se comenzó a observar desde 1960 en los Centros de Madres, en las Agrupaciones de Artesanas y en Asociaciones Mixtas. Los Centros de Madres fueron las primeras organizaciones de mujeres, impulsadas por el Estado y con objetivos surgidos externamente, asumidas por las comunidades aymaras para obtener beneficios sociales y lograr adaptarse al régimen administrativo político-social imperante. Las Agrupaciones de Artesanas son el fenómeno organizativo femenino con mayor importancia, las que se inician gracias a ONG y en donde la:

(...) generación de ingresos familiares vía producción y comercialización de artesanías textiles, aumento del nivel de información y capacitación que permita mejorar sus actuales condiciones de vida, son los fundamentos que guían estas iniciativas organizativas (...) (Carrasco 1993a:190).

Finalmente, las organizaciones mixtas nacen de manera sustancial en las zonas urbanas y son en lo principal definidas como Centros de Hijos de Pueblos, Organizaciones Étnicas-reivindicativas y Asociaciones de Artesanos (Ibíd. Pp. 191).

Al promulgarse la Ley Indígena $\mathrm{N}^{\circ} 19.253$ en 1993, se plantea que los pueblos originarios reconocidos por el Estado mantienen un nuevo tipo de regulación de tipo organizacional. Este se basa en una ampliación de la figura tradicional de la comunidad a una más funcional y deslocalizada, como las Comunidades y las Asociaciones Indígenas. La primera de ellas posee un carácter territorial a diferencia de la segunda que tiene que ver con aspectos funcionales abarcando diversos objetivos (educacionales, económicos, culturales, entre otros). Es importante resaltar que el 2009 entra en vigencia el Convenio 169 de la Organización Internacional del Trabajo (OIT), el que reconoce la existencia de diferentes modos de vida, tradiciones y costumbres de los pueblos indígenas, así como también de instituciones y leyes consuetudinarias que reconocen la presencia de una fricción interétnica y garantizan la reivindicación de especificidades culturales de los grupos indígenas.

En la región de Arica y Parinacota existen 69 Comunidades y 142 Asociaciones Indígenas, en las que hay 5.903 integrantes. Específicamente, las Asociaciones Indígenas con los años han mostrado un incremento significativo en su cantidad, primando siempre las organizaciones mixtas y observándose una participación gradual de las mujeres en ellas.

De acuerdo con el carácter de esta investigación, así como también de los objetivos que sustentan la misma, la metodología es de tipo cualitativa. En este sentido, se efectuó una revisión y análisis de información secundaria relacionada a la histórica participación de las mujeres en los espacios públicos. Se realizaron fichas descriptivas que nos permitieron organizar los aspectos centrales de cada temática así como también analizar la importancia que recae en cada una de estas aristas respecto de la participación femenina. Finalmente, se llevó a cabo el análisis y sistematización de información empírica por medio del análisis de 20 entrevistas estructuradas a mujeres aymaras que participan en las 5 Asociaciones Indígenas femeninas vigentes y activas, estas se inscriben bajo diversos objetivos (educacionales, culturales, profesionales comunes a sus miembros y económicos). De esta manera, las organizaciones elegidas permiten visualizar distintos motivos de participación y, por tanto, diferentes valoraciones que las mujeres le entregan a estas Asociaciones.

\section{Participación y experiencia de las mujeres aymaras en las Asociaciones Indígenas Femeninas}

En primer lugar, resulta necesario conocer los objetivos organizativos que salen a la luz en los discursos de las mujeres participantes de las Asociaciones Indígenas consideradas en este estudio, que permitirán entender mejor la creación, desarrollo y experiencias de estas instancias femeninas.

A pesar de la amplia gama de opiniones rescatadas, se percibe de manera indiscutida una finalidad predominante relacionada con la reivindicación de la cultura aymara frente a la sociedad chilena mestiza. Con ello se presenta claramente el deseo de mantener esta práctica asociativa femenina, manifestándose por parte de las integrantes del grupo, de manera constante, la idea de un cambio en las prácticas culturales tradicionales que permita la generación de un espacio social reconocido no excluyente hacia las mujeres. Y, de esta forma, agrupar a las mujeres y promover en ellas la búsqueda de un espacio anteriormente no alcanzado. Así, el 
tema de la necesidad o importancia que las mujeres tengan una participación sociopolítica activa, que antes no se daba, aparece como una razón de peso para mantenerse unidas en la organización.

\section{(...) En el tema de ganar espacios porque como te decía es un poco machista la cultura o sea los hombres (...) han sido pocas las mujeres que han sido dirigentes (...) de renombre, han sido los hombres entonces (...) un poco de ganar espacios, de ir incidiendo (...) (Entrevistada $\mathrm{N}^{\mathrm{o}} 10$ ).}

Mediante las entrevistas realizadas, y en relación con la importancia que le atribuyen a la participación de la mujer en las Asociaciones, se concluye que existe un estímulo por parte de ellas que ha demostrado ser eficaz en la temática; y este estímulo hoy está dado en poder tener las mismas posibilidades de acceder a cargos dirigenciales que -antiguamente- solo pertenecían a los hombres. Esto ha tomado fuerza, generando un liderazgo femenino que va en aumento permitiendo que hayan perdido el miedo a ser recriminadas. Han alcanzado mayor fuerza en temas de liderazgo a nivel organizacional, entregando una mirada diferente frente a propuestas políticas y participativas de las autoridades por medio de la ejecución de proyectos y actividades socioculturales generando nuevos canales de participación que antiguamente eran inexistentes e impensados, debido a las características en que tradicionalmente se distribuían los roles para cada género en la cultura aymara.

De esta manera, han adquirido un reconocimiento por parte de la sociedad en general por su perseverancia, esfuerzo y dedicación y, específicamente, por las mujeres aymaras quienes muchas veces aceptan los liderazgos femeninos y la participación de las mujeres en organizaciones, pero por diferentes motivos no son partícipes de estas Asociaciones y menos aún de cargos de representación pública:

\section{(...) Algunas veces algunas personas lo aceptan (...) porque es mejor porque la mujer tiene que crecer, tiene que decir lo que siente, sacar los derechos de uno y luchar por lo que uno quiere (...) otras mujeres no lo aceptan, otras mujeres se callan (...) se callan pero ellas quieren hacer eso pero no pueden (...) (Entrevistada $\mathrm{N}^{\mathrm{o}} 8$ ).}

Un aspecto importante de considerar en el desarrollo y permanencia de las mujeres aymaras en estas agrupaciones es el apoyo entregado por el núcleo familiar desde su ingreso a este tipo de participación. Contando con este apoyo de sus parejas, sus madres y sus hijos en la realización de las tareas domésticas cotidianas, se pudo constatar que el respaldo familiar proporciona ayuda, confianza y un mejor desempeño en la integración en este tipo de organizaciones.

Sin embargo, con el correr de los años se presentan también dificultades en el desarrollo de estas organizaciones. De manera general, se puede entender que los desacuerdos se establecen bajo dos perspectivas. A nivel macro, las discrepancias establecidas con los servicios públicos obstaculizando los voluntariados y charlas realizadas por las organizaciones femeninas para informar masivamente acerca de los contenidos de género gubernamentales presentan importantes limitantes que no permiten su plena participación:

Las mujeres fueron muy atacadas por los servicios públicos (...) después vino una decadencia por esta misma persecución, los servicios públicos se llaman y empezaron a cortarles las alas po' (...) (Entrevista $\left.\mathrm{N}^{\circ} 1\right)$.

Además, la competencia existente entre las diferentes Asociaciones, así como también los intereses individuales mostrados por algunas dirigentes, principalmente por la permanencia en los cargos directivos -situación que resulta bastante comúnson temas que salen a la luz en la mayoría de las respuestas entregadas. Sin embargo, esto último resulta muchas veces contradictorio, ya que, al ponerse a disposición los cargos directivos en las organizaciones, sus socias no muestran interés por aceptar los puestos, ya sea por motivos de tiempo, por la inexistencia de remuneración económica, etc.

\section{(...) Intereses creados de algunas dirigentes,} entonces quieren ser ellos no más lo (...) estar ellos, entonces por ejemplo si eligen un presidente este año siempre son dos años los que dura la presidencia, hay que renovar, y siempre tiene que ser el mismo, el mismo (...) (Entrevista $\mathrm{N}^{\mathrm{o}} 16$ ).

Por otra parte, a nivel micro las discrepancias de opinión dentro de las Asociaciones se 
presentan con frecuencia por diversos motivos, como por ejemplo la diferencia etaria, lo que crea opiniones desiguales y un clima hostil que genera desunión y que alimenta los desacuerdos dentro de las integrantes. Por otra parte, la participación dentro de estas agrupaciones condiciona el acceso al mercado laboral:

\section{(...) Ha tenido un costo y sacrificios por ejemplo postergar a mi familia, yo no tra- bajo (...) yo como dirigente social o estar en algunos temas que han sido candentes en esta región me coartan la posibilidad de trabajo en cualquier lao' (...) ese es el costo que uno paga, y yo al no tener trabajo hay solamente un ingreso que es mínimo entonces tengo lo justo y necesario no ma' (...) (Entrevistada $\mathrm{N}^{\mathrm{o}} 1$ ).}

De esta forma, del total de entrevistas realizadas, se antepone una visión crítica de su participación en las Asociaciones. Los testimonios coinciden en destacar el carácter funcional de estas Asociaciones en donde los objetivos que se enmarcan dentro de la agrupación pierden su rumbo al dejarse llevar por fines personales de sus integrantes. De esta manera, las dirigentes o socias responsables de elaborar y gestionar los proyectos, en muchas ocasiones obtienen beneficios individuales de tipo económico. Así, las organizaciones al tener acceso a distintos beneficios otorgados por el gobierno y al obtener recursos para ciertas acciones que se encuentran -o vienen- definidas, muchas veces desvirtúan los objetivos por los que se crea la Asociación. Además, se sostiene que las organizaciones aunque sean apoyadas desde el Estado, pueden no ser proactivas y manifestar poca capacidad de gestión:

\section{(...) Son funcionales aún están sometidas al paternalismo, al asistencialismo, no tienen capacidad de crecimiento, son or- ganizaciones que por ejemplo no buscan el bien común (...) si hay algo individual allá atacan (...) (Entrevistada $\left.\mathrm{N}^{\circ} 1\right)$.}

Finalmente, según la información obtenida salen a la luz expectativas futuras en cada Asociación. Estos anhelos se representan por la búsqueda de mayor independencia de las asociadas; postulación a programas privados y estatales que le permitan ejecutar o llevar a cabo proyectos de promoción organizativa, productivos, etc.; realización de actividades de capacitación o formación en temáticas como género y violencia; aperturas de nuevos mercados, nacionales e internacionales, para -por ejemplo- la venta de la producción artesanal textil y continuar con la herencia cultural, sea en el aspecto del rescate y mantención de la tradición textil:

\section{(...) Mira todas las señoras todas quieren seguir pero (...) expectativas buenas no (...) se está conversando algo de intentar innovar más el producto (...) de ya han inventao' rayitas, antes hacían puro liso (...) (Entrevistada $\left.\mathrm{N}^{\circ} 5\right)$.}

Por otro lado, el traspaso de su experiencia mediante actividades, promover en otras organizaciones la importancia del trabajo colectivo y de la participación de las mujeres, es otro aspecto significativo que piensan seguir realizando:

\section{(...) El tema de derecho, el tema político $y$ otras instancias para poder llevar un tema diferente y así poder llevarlos a otras organizaciones que es lo que intentamos hacer, hacer talleres en otras organiza- ciones, en otras ciudades, otras regiones a nivel nacional de poder apoyar (...) (Entrevistada $\mathrm{N}^{\mathrm{o}} 10$ ).}

Sin duda, dentro de este ámbito se percibe un objetivo común que enfatiza el crecimiento íntegro de la organización, comprendiendo a fin de cuentas que en la medida que el desarrollo se genere a partir de cada uno de los integrantes, llevará indiscutidamente a un eficaz trabajo grupal.

\section{Reflexiones finales}

Frente a lo expuesto, es posible concluir que las organizaciones de mujeres aymaras en el espacio público permitieron la socialización e intercambio social que no mantenían tradicionalmente, buscando la oportunidad de sacar a la luz aspectos y temáticas del interés colectivo femenino aymara. Resalta la importancia atribuida a la protección y permanencia de patrones culturales aymaras, en donde por medio del traspaso de tradiciones y costumbres buscan la entrega de nuevos conocimientos y establecer la continuidad cultural. Del mismo 
modo, la mujer aymara ha logrado desempeñarse en una variedad de espacios sociales y temáticas que en cierta medida eran lejanos y genéricamente inaccesibles, como es la política y los derechos de la mujer. Sin embargo y, a pesar del valor que posee la reinvindicación cultural aymara mediante estas instancias públicas, señalan que muchas veces la finalidad organizacional pierde valor al entrelazarse con fines individuales que desprestigian el largo camino que han traido consigo por años.

A pesar de ello, con los años se ha experimentado un aumento progresivo en la participación de las mujeres aymaras en las Asociaciones Indígenas, en donde cualidades como el liderazgo y nuevas actitudes dirigenciales han servido como referentes para la reivindicación femenina aymara en la búsqueda de nuevos horizontes.

\section{Agradecimientos}

Este documento forma parte del Proyecto UTA Mayor 2016; como asimismo, cuenta con el apoyo del Convenio de Desempeño Universidad de Tarapacá-Mineduc. De igual forma, agradecemos las sugerencias aportadas por los evaluadores, que sin duda han ayudado a mejorar este artículo.

\section{Referencias Citadas}

Aguirre C., I.

2003 "Participación política y social de mujeres indígenas: El caso de una lideresa tradicional". Revista Cuicuilco 10:27. Escuela Nacional de Antropología e Historia (ENAH). Distrito Federal, México.

Azaola, Elena

2012 "La violencia de hoy, las violencias de siempre". Revista Desacatos 40: 13-32, Distrito Federal, México.

Bidaseca, K.

2010 Perturbando el texto colonial. Los Estudios (Pos) coloniales en América Latina. Editorial SB, Buenos Aires.

Bonfil, $\mathrm{P}$.

2002 "Las mujeres indígenas y su participación política: un movimiento contra la desmemoria y la injusticia". En Participación política de las mujeres y gobiernos locales en México, Editado por Dalia Barrera Bassols, pp. 67-98. GIMTRAP, México.

Bouteldja, $\mathrm{H}$.

2014 “'Feministas o no? Pensar la posibilidad de un 'feminismo decolonial'", con James Baldwin y Audre Lorde. En Tabula Rasa, 21: 77-89, España.

Calfio, M. y Velasco, L.

2005 Mujeres indígenas en América Latina: ¿Brechas de género o de étnia? Seminario internacional Pueblos Indígenas y Afrodescendientes de America Latina y el Caribe: Relevancia y Pertinencia de la Información Sociodemográfica para Políticas y Programas. CEPAL, Santiago, Chile.

Carrasco, A. M.

1993a "Mujeres y participación social en la sociedad aymara contemporánea". En Huellas, Seminario Mujer y Antropología, CEDEM, pp. 175-183, Santiago, Chile.

1993b Antecedentes para un Diagnóstico de la Situación de la Mujer en el Sector Rural de la I Región de Tarapacá. Documento de trabajo, Unidad de la Mujer, Taller de Estudios Andinos, Arica, Chile.

1998 Mujer rural y participación social en la I Región de Tarapacá. Borrador de documento. Norte Mujer (Manuscrito), Arica, Chile.

2006 Hacia un nuevo significado de la política: Los movimientos de mujeres en la Región de Tarapacá, Chile
(1910-1973). Tesis Doctoral en Historia presentada en la Universitat de Barcelona, Facultat de Geografía i Història, Departamentd' antropología i HistòriaD' Amèrica y Àfrica; Programa de Doctorat Recuperació de la Memòria. AmèricaLlatina (1998-2000) Barcelona, España.

De Barbieri, T.

1992 "Sobre la categoría género. Una introducción teóricometodológica". En Ediciones de las Mujeres, pp. 17:111-128, Santiago, Chile.

De Oliveira, O. (Coordinadora)

1989 Trabajo, poder y sexualidad. Programa Interdisciplinario de Estudios de la Mujer. El Colegio de México, México.

Fassler, C.

2003 Desarrollo y participación política de las mujeres. Instituto Nacional Demócrata (IND), Ganando con las mujeres. Fortaleciendo los partidos políticos. III Conferencia Internacional de la Red de Estudios sobre el Desarrollo Celso Furtado, Buenos Aires, Argentina.

Frohman, A. y Valdés, T.

1993 Democracy in the Country and in the Home: The Women's Movement in Chile. Working Paper, Serie Estudios Sociales, Flacso, Santiago, Chile.

Gavilán, V.

1993 Antecedentes para comprender la violencia intrafamiliar entre los aymaras del Norte de Chile. En Manual de violencia intrafamiliar. Servicio de Salud Arica y Servicio Nacional de la Mujer, Arica, Chile.

Gaviola, E.

1988 Chile. Centros de Madres. ¿La mujer popular en movimiento? En Nuestra memoria, nuestro futuro. Mujeres e historia, Ediciones de las mujeres No 10, Grupo Condición femenina-CLACSO, Santiago, Chile.

1994 "Movimiento de mujeres en Chile (1912-1978)". Revista Proposiciones 24:7, Ediciones Sur, Santiago, Chile.

González, H.

1995 Los migrantes aymaras en la ciudad: Acceso a educación, vivienda y salud. Serie Documentos de Trabajo. Corporación Norte Grande, Arica, Chile.

1996 Características de la migración campo-ciudad entre los aymaras del norte de Chile. Serie Documentos de Trabajo. Corporación Norte Grande, Arica, Chile. 
González, H.; Tabilo, K. y Venegas, F.

1995 Las agrupaciones de residentes aymara urbanos en el norte de Chile: adaptación a la ciudad y vínculos con las comunidades de origen. Serie Documentos de Trabajo, Corporación Norte Grande, Arica, Chile.

Gundermann, $\mathrm{H}$.

2001 "Procesos regionales y poblaciones indígenas en el norte de Chile: Un esquema de análisis con base en la comunidad y los cambios de la comunidad andina". Revista Estudios Atacameños 21:89-112, Universidad Católica del Norte, San Pedro de Atacama, Chile.

2003 "Sociedades indígenas, municipio y etnicidad: La transformación de los espacios políticos locales andinos en Chile". Revista Estudios Atacameños 25:55-77, Universidad Católica del Norte, San Pedro de Atacama, Chile.

\section{Grebe, M.E.}

1981 “Cosmovisión aymara”. Revista de Santiago 1: 61-79, Santiago, Chile.

Hernández, A.

2000 "Entre el etnocentrismo feminista y el esencialismo étnico. Las mujeres indígenas y sus demandas de género". Revista Debate Feminista 24:206-229, Ciudad de México.

Kirkwood, J.

1990 Ser política en Chile: los nudos de la sabiduría feminista. Cuarto propio, Santiago, Chile.

Lamas, $\mathrm{M}$.

1999 "Usos, dificultades y posibilidades de la categoría género". Revista Papeles de Población 21:147-178, Universidad Autónoma del Estado de México Toluca, México.

Luna, L.

2004 Los movimientos de mujeres en América Latina y la renovación de la historia política. Libro electrónico editado e impreso por fem-e-libros, México.

Meloni, C.

2012 Las fronteras del feminismo. Teorías nómadas, mestizas y posmodernas. Editorial Fundamentos, Madrid, España.

Molyneux, M.

2001 "Género y ciudadanía en América Latina: cuestiones históricas y contemporánea”. En Debate Feminista, pp. 12-23, México.
Montecino, S.

2004 "Hacia una antropología del género en Chile". En Mujeres, espejos y fragmentos. Antropología del género y salud en el Chile del Siglo XXI. Sonia Montecino, René Castro, Marco Antonio de la Parra (Comp.), Santiago, Chile.

Moore, $\mathrm{H}$

1991 Antropología y feminismo. Instituto de la mujer, Universidad de Barcelona. Ediciones Cátedra, Madrid, España.

Ruiz, S. y Bonometti, P.

2010 "Las mujeres en América Latina: Indicadores y datos". Revista Ciencias Sociales 126-127:75-87, Universidad de Costa Rica, Costa Rica.

Safa, $\mathrm{H}$.

2008 "Igualdad en diferencia: género y ciudadanía entre los indígenas y afrodescendientes". En Feminismos en la Antropología: nuevas propuestas críticas. L. Suárez, E. Martín, R. Hernández (Coordinadoras), pp. 55-79. Ponencias presentadas en el XI Congreso de Antropología: retos teóricos y nuevas prácticas, Donostia-San Sebastián.

Scott, J.

1996 "El género: una categoría útil para el análisis histórico". En El género. La construcción cultural de la diferencia sexual, editado por M. Lamas, pp. 265-302. PUEG/ Grupo Editorial Miguel Ángel Porrúa, Universidad Nacional Autónoma de México, México.

Valdés, T.

2004 Hacia una agenda sobre sexualidad y derechos humanos en Chile. FLACSO, Centro Latinoamericano, Santiago, Chile.

Van Kessel, J.

1993 Pachamama, La Virgina: la que Creó el Mundo y Fundó el Pueblo. CIDSA, Puno.

Zúñiga, M.

2014 "Las mujeres en los espacios públicos: entre la violencia y la búsqueda de libertad". Región y Sociedad 4:77-100, México. 